\title{
Estudio descriptivo del color de manto y señas del perro Ovejero Magallánico, Chile
}

\author{
Barrios, N. ' ; Bórquez, A. ' ; Gómez, M. ${ }^{\circledR}$; Tafra, V. ${ }^{1}$ y Sponenberg, P. ${ }^{3}$
}

'Facultad de Recursos Naturales y Medicina Veterinaria. Universidad Santo Tomás. Sede Puerto Montt. Puerto Montt. Chile.

${ }^{2}$ Instituto de Farmacología y Morfofisiología. Facultad de Ciencias Veterinarias. Universidad Austral de Chile. Campus Isla Teja. Valdivia. Chile. ${ }^{3}$ Department of Biomedical Sciences and Pathobiology. Virginia-Maryland College of Veterinary Medicine. Virginia Tech. Blacksburg. VA. USA.

\section{PALABRAS CLAVE ADICIONALES}

Ovejero magallánico.

Color de manto.

Eumelanina.

Feomelanina.

\section{RESUMEN}

El Ovejero Magallánico constituye una raza canina no reconocida oficialmente, localizada en el extremo sur de Chile en la Región de Magallanes y Antártica Chilena. El objetivo de este estudio fue realizar una primera descripción de la coloración del manto y señas del perro Ovejero Magallánico en una muestra de 74 caninos adultos. Los resultados de este estudio descriptivo preliminar indicaron el predominio del pigmento eumelanina en muestras de pelo de perros Ovejeros Magallánicos de la zona Austral de Chile. Se determinó la presencia de 16 fenotipos de colores de manto, predominando las coloraciones oscuras como gris pizarra, gris pizarra tricolor, azul mirlo y negro. Además, se observaron 3 colores de manto (marrón/arena, negro/marrón y arena azulado tricolor) no descritos para razas Collies. Se determinaron 12 fenotipos de señas en el manto del perro Ovejero Magallánico, observándose 2 patrones de señas (antifaz y orejas) que no se presentan en otras razas Collies. El perro Ovejero Magallánico, presentó algunas similitudes en coloración de manto y señas al ser comparadas con las razas Bearded Collie, Bobtail y Border Collie. Sin embargo, las tonalidades dentro y entre los colores observadas en el manto del Ovejero Magallánico evidencian un aspecto matizado, diferenciándolo de las razas Collie tradicionales.

\section{Descriptive study of coat colour and marking in Ovejero Magallanico dogs, Chile}

\section{SUMMARY}

The Ovejero Magallanico is a non-officially recognized dog breed located in southern Chile's Magallanes and Chilean Antarctica Region. The aim of this study was to conduct an initial coat colour and markings description in a 74 adult Ovejero Magallanico dogs sample. The results of this preliminary descriptive study indicated a eumelanin pigment predominance in hair samples collected from Chilean Austral area Ovejero Magallanico dogs. A total of 16 different color coat phenotypes were observed with predominance of dark colours such as grays, blue merle and black. In addition, 3 observed coat colour patterns (brown and tan; black tricolor, brown tricolor, and blue tricolor) are not described for the Collie dog breed. Twelve patterns of markings in the coat of Ovejero Magallanicos were determined. Two marking patterns (mask and ears) that are not present in Collies were observed in Ovejero Magallanico dogs. Ovejero Magallanico dogs have some similarities in coat colour and markings with Bearded Collies, Border Collies and Bobtails. However, the colour tones within and between the coat colour patterns seen in Ovejero Magallanico dogs showed distinct features from other traditional Collie dog breeds.

\section{INFORMACIÓN}

Cronología del artículo.

Recibido/Received: 22.07.2015

Aceptado/Accepted: 11.01 .2016

On-line: 16.03 .2016

Correspondencia a los autores/Contact e-mail:

marcelogomez@uach.cl

\section{INTRODUCCIÓN}

El Ovejero Magallánico constituye una raza canina no reconocida oficialmente, localizada en el extremo sur de Chile en la Región de Magallanes y Antártica Chilena. Esta raza se originó de perros ovejeros británicos llegados a la zona austral de Chile a fines de 1800 y comienzos de 1900 (Fuenzalida, 2006). Registros del Border Collie Museum de Inglaterra, indican que las razas caninas que podrían haber sido introducidas y utilizadas en la región de Magallanes durante ese período incluirían al Old Wesh Grey, raza británica extinta que dio origen a Bearded Collie, el Bobtail, el Border Collie, o alguna otra variedad de Collie (Fuenzalida, 2006). El perro Ovejero Magallánico es una raza canina de pastoreo que cumple la función de mantener al rebaño ovino agrupado y facilitar su arreo (Fuenzalida, 2006). Las características morfológicas o 
fenotípicas asociadas a un perro Ovejero Magallánico, de acuerdo a los criterios definidos por los ganaderos locales, incluyen un perro de tamaño mediano, presencia de pelaje largo o semilargo, orejas caídas o semierguidas y que se encuentre en trabajo activo en estancias ovinas (Tafra et al., 2014). Estudios preliminares indican índices zoométricos que lo clasifican como dolicocéfalo, dolicotorácico, longilíneo y braquipélvico (Tafra, 2012). El objetivo de este estudio fue realizar una primera descripción de la coloración del manto y señas del perro Ovejero Magallánico en una muestra de caninos adultos.

\section{MATERIAL Y MÉTODOS}

\section{MATERIAL BIOLÓGICO}

Se realizó un muestreo dirigido de 74 perros adultos, con un rango de edad de 1 a 10 años, cuyo fenotipo cumpliera con las características morfofuncionales asociadas a un perro Ovejero Magallánico. Los animales provenían de la Región de Magallanes y Antártica Chilena, específicamente de estancias ovinas de las provincias de Magallanes, Última Esperanza y Tierra del Fuego. Muestras de pelo fueron extraídas manualmente a nivel de la región interescapular (lugar representativo del color predominante en el cuerpo) y fueron conservados en bolsas herméticas para posterior análisis microscópico (Dreger y Schmutz, 2010). Adicionalmente, se obtuvo un registro fotográfico del cuerpo completo de cada ejemplar con el fin de evaluar la distribución de color de manto y señas.

\section{ANÁLISIS DE MUESTRAS Y ANIMALES}

Las muestras de pelo fueron analizadas por microscopia óptica digital (Microscopio Motic BA310 serie Biological Microscope) para evaluar la presencia de pigmentos melanocíticos (eumelanina y feomelanina). Luego, las muestras de pelo fueron fijadas en un portaobjeto con cinta adhesiva y se obtuvieron fotografías digitales de su base, centro y ápex en aumentos de $4 \times-10 \times$. Posteriormente, se comparó la pigmentación del pelo con la fotografía del manto completo de cada perro. Para la clasificación de color de manto y señas se utilizó como base los colores de manto y las señas blancas aceptadas por la Federación Cinológica Internacional (FCI) para las razas Bearded Collie, Bobtail y Border Collie y la descripción reportada en el estudio zoométrico del Ovejero Magallánico realizado por Tafra et al. (2014).

\section{RESULTADOS Y DISCUSIÓN}

En relación a los pigmentos predominantes en las muestras de pelo de 74 perros Ovejeros Magallánico en estudio, se observó una mayor frecuencia de presentación del pigmento eumelanina (54,8\%), seguido de feomelanina $(27,8 \%)$ y ausencia de pigmento $(11,1 \%)$. No se observaron diferencias de frecuencia de pigmentos en relación a diferentes edades. Cabe mencionar que existió un 6,3\% de muestras que fueron clasificadas como sin dominancia debido a que no presentaron una frecuencia marcada hacia ningún pigmento. Al analizar la distribución de frecuencias de pigmentos en la base,
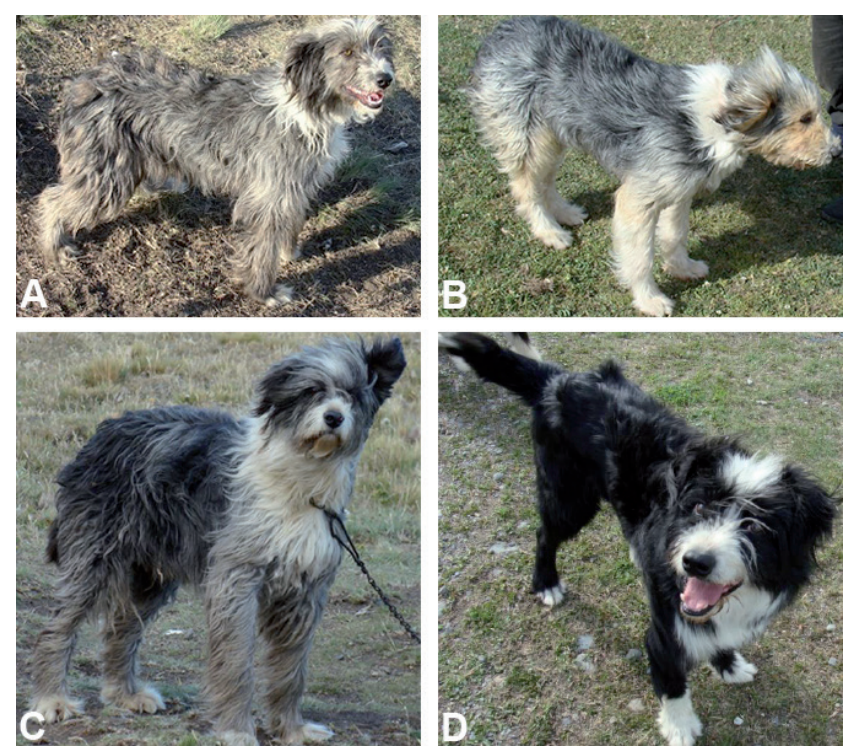

Figura 1. Fotografías de los principales fenotipos de color de manto observados en 74 perros Ovejeros Magallánicos. Gris pizarra (A); Gris pizarra tricolor (B); Azul mirlo (C); Negro con o sin blanco (D) (Photographs of the main coat color phenotypes observed in 74 dogs Ovejero Magallanico dogs. Grey (A); Grey tricolor (B); Blue merle (C); Black with or without white (D)).

centro y ápex de los pelos de 74 perros Ovejeros Magallánicos, se observó un 39,7\% de eumelanina en la base del pelo seguido de ausencia de pigmentos (34,9\%) y feomelanina (25,4\%). En el centro del pelo predominó el pigmento eumelanina $(73,0 \%)$, seguido por el pigmento feomelanina $(24,6 \%)$ y ausencia de pigmento $(2,4 \%)$. En ápex del pelo también predominó el pigmento eumelanina $(42,1 \%)$, seguido por el pigmento feomelanina $(38,9 \%)$ y ausencia de pigmento $(19,0 \%)$. En relación a la presentación de coloraciones dentro de un mismo pelo, se evidenció que un $27,0 \%$ de los pelos presentaron coloración mixta de eumelanina y feomelanina, un 25,4\% eumelanina y sin pigmentación, un $18,3 \%$ eumelanina de distribución completa, un 13,5\% de feomelanina y sin pigmentación, un 7,1\% eumelanina, feomelanina y sin pigmentación; y feomelanina completo, respectivamente y un 1,6\% sin pigmentación de forma completa. El color de la capa en caninos esta determinado por la densidad y distribución de los tipos de melanina (eumelanina, feomelanina) en los folículos pilosos (Hoekstra, 2006). En mamíferos, los genes que regulan la síntesis de eumelanina y feomelanina son el Mc1r (Melanocortin 1 receptor) y el Agouti (Ito, 2003). Se determinaron un total de 16 fenotipos de colores de manto, observándose con mayor frecuencia el color gris pizarra $(18,9 \%)$, gris pizarra tricolor (10,8\%), azul mirlo (10,8\%) y negro (9,5\%) (figura 1). El gris pizarra es considerado una modificación del fenotipo negro y en ambos el gen más común para la presentación es $\beta$-defensin (Candile et al., 2007). Gris pizarra tricolor es una modificación del marrón/arena con participación del gen Agouti y el azul mirlo es el resultado de la expresión del locus SILV un gen dominante (Clark et al., 2006). Al comparar con la raza Bearded Collie, se observó similitudes en los colores gris pizarra, negro y tonalidades de arena (FCI, 2010). La raza Bobtail, quien presenta colores de manto en 
A)
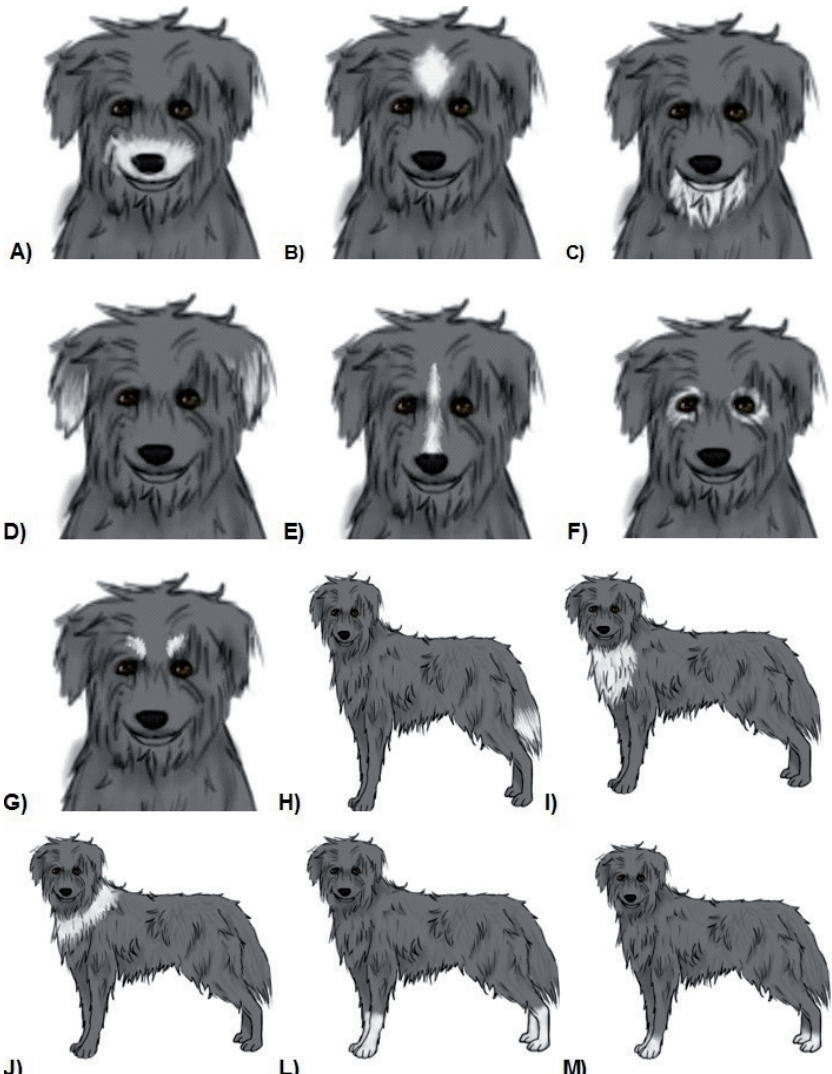

L)

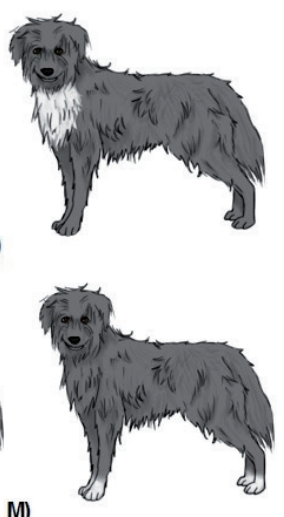

Figura 2. Representación esquemática de los 12 fenotipos de señas observadas en 74 Ovejeros Magallánicos. Hocico (A); Moño (B); Barba (C) Orejas (D): Sagital (E); Antifaz (F); Cejas (G); Punta de cola (H); Pecho (I); Collar (J); Botin alto (L); Botin bajo (M) (Schematic view of the 12 marking pattern phenotypes observed in 74 Ovejero Magallanico dogs. Muzzle spotting (A); Star spotting (B); Beard spotting (C) Ears spotting (D) Stripe spotting (E); Mask spotting $(\mathrm{F})$; Eyebrows spotting $(\mathrm{G})$; Tail tip $(\mathrm{H})$; Chest (I); Necklace $(\mathrm{J})$; High socks (L); Low socks (M)).

cualquier tonalidad de gris, grisáceo y azul, se determinó que el Ovejero Magallánico presenta similitudes con el Ovejero Magallanico en los colores de manto negro y azul. Sin embargo, el Ovejero Magallánico presentó tonalidades mixtas en las zonas unicolores, las cuales no son aceptadas para la raza Bobtail (FCI, 2011). La raza Border Collie puede presentar mantos de cualquier color y sus variaciones, sin embargo el color blanco no debe prevalecer, característica que si se observó en el Ovejero Magallánico (FCI, 2010). Este estudio preliminar indica que el Ovejero Magallánico presenta coloraciones de manto similares con las razas Collie con ancentro común, no obstante, presenta coloraciones (color marrón y arena, negro y marrón y arena azulado tricolor) no descritas en las razas Collies anteriormente señaladas. Se determinaron 12 señas en el manto de 74 perros Ovejeros Magallánicos (figura 2). Las señas predominantes se observaron en el hocico $(63,5 \%)$, barba $(58,1 \%)$, moño $(44,6)$ punta de cola y orejas con un $40,5 \%$. Las señas presentaron semejanzas con las señas blancas aceptadas en la raza Bearded Collie, ya que ambos exhiben un parche en la parte más dorsal de la cabeza denominada moño según Tafra et al. (2014) y estrella para la raza Bearded Collie (FCI, 2010). Ambas razas presentan señas blancas en hocico, cejas, punta de cola, pecho y collar. En el perro Bearded Collie no se acepta el blanco por sobre las articulaciones tibio-tarsianas (FCI, 2010). Sin embargo, en el perro Ovejero Magallánico se observó tanto el botín bajo (seña bajo la articulación tibio-tarsiana) como el botín alto (seña sobre la articulación tibio-tarsiana) (Tafra et al., 2014). En el Border Collie, la FCI acepta cualquier tipo de señas, sin identificar cada una de ellas, siendo el único requisito que no exista predominio del blanco por sobre el color de manto (FCI, 2010). Finalmente, al comparar el perro Ovejero Magallánico con la raza Bobtail se evidenció similitud en un tipo de seña, la cual se puede ubicar en la parte baja de los miembros posteriores en la raza Bobtail denominada calcetines (FCI, 2011) y que en el Ovejero Magallánico se denomina botín bajo (Tafra et al., 2014). Sin embargo, en el Ovejero Magallánico esta seña se puede presentar tanto en los miembros anteriores como posteriores (Tafra et al., 2014). Se observaron dos señas descritas para el Ovejero Magallánico (antifaz y orejas) que no están presentes en otras razas Collies. Finalmente los resultados obtenidos en el presente estudio complementan la investigación zoométrica realizada por Tafra et al. (2014), que en conjunto con la descripción del manto y señas permiten señalar que el perro Ovejero Magallánico difiere de las razas Collie actualmente existentes reconocidas por la FCI, pero con las cuales comparten un ancestro común. Sin embargo, este es un estudio preliminar y se necesitan estudios posteriores para correlacionar los resultados de color de manto con análisis genéticos moleculares.

\section{BIBLIOGRAFÍA}

Candille, S.I.; Kaelin, C.B.; Cattanach, B.M.; Yu, B.; Thompson, D.A.; Nix, M.A.; Kerns, J.A.; Schmutz, S.M.; Millhauser, G.L. and Barsh, G.S. 2007. A defensin mutation causes black coat color in domestic dogs. Science, 318: 1418-1423.

Clark, L.A.; Wahl, J.M.; Rees, C.A. and Murphy, K.E. 2006. Retrotransposon insertion in SILV is responsible for merle patterning of the domestic dog. Proc Natl Acad Sci, 31: 1376-1381.

Dreger, D.L. and Schmutz, S.M. 2010. A new mutation in MC1R explains a coat color phenotype in 2 old breeds: Saluki and Afghan Hound. $J$ Hered, 101: 644-649.

Federación Cinológica Internacional (FCI). 2010. Collie Barbudo (Bearded Collie). Estándar- $\mathrm{FCl}$ (271).

Federación Cinológica Internacional (FCI). 2011. Antiguo Perro Inglés (Old English Sheepdog). Estándar- $\mathrm{FCl}$ (16).

Fuenzalida, Á. 2006. El perro Ovejero Magallánico; testimonio de reconstrucción histórica. (Tesis de Licenciatura en Historia). Pontificia Universidad Católica. Santiago. Chile.

Hoekstra, P.W. 2006. Genetics, development and evolution of adaptive pigmentation in vertebrates. Heredity, 97: 222-234.

Ito, S. and Wakamatsu, K. 2003. Quantitative analysis of eumelanin and pheomelanin in humans, mice, and other animals: a comparative review. Pigment Cell Res, 16: 523-531.

Tafra, V. 2012. Caracterización zoométrica y faneróptica del perro Ovejero Magallánico. Memoria de título. Facultad de Ciencias Veterinarias, Universidad San Sebastián. Puerto Montt. Chile.

Tafra, V.; Barrios, N.; Godoy, J.; De la Barra, R. y Gómez, M. 2014. Primera caracterización morfoestructural y faneróptica del perro Ovejero Magallánico. Chile. Arch Zootec, 63: 371-380. 
